\title{
Cancer Survivor Study (CASUS) on colorectal patients: longitudinal study on physical activity, fitness, nutrition, and its influences on quality of life, disease recurrence, and survival. Rationale and design
}

\author{
Luisa Soares-Miranda ${ }^{1}$ (D) Sandra Abreu ${ }^{1}$ - Marco Silva ${ }^{2}$ - Armando Peixoto ${ }^{2}$. \\ Rosa Ramalho $^{2}$ - Pedro Correia da Silva ${ }^{3}$ - Carla Costa ${ }^{4,5}$ - João Paulo Teixeira ${ }^{4,5}$. \\ Carla Gonçalves $^{6} \cdot$ Pedro Moreira $^{1,6} \cdot$ Jorge Mota $^{1} \cdot$ Guilherme Macedo $^{2}$
}

Accepted: 27 September 2016

(C) Springer-Verlag Berlin Heidelberg 2016

\begin{abstract}
Purpose Evidence suggests that being physically active in combination with a healthy diet contributes to diminish colorectal cancer risk. However, if this is true for colorectal cancer primary prevention, the same is not clear for its recurrence after colorectal cancer treatments. Data on cancer survival are scarce, and there is a need for greater attention on these survivors' lifestyle behavior. This manuscript describes rationale and design of the Cancer Survival Study (CASUS) on colorectal patients, a longitudinal observational study with the aim of investigating how physical activity, physical fitness, and dietary intake are related with their quality of life, disease recurrence, and survival.

Methods The CASUS on colorectal patients is a longitudinal cohort study on colorectal survivors, aged 18 years or older, recruited 6, 12, and 24 months after surgery. Upon recruitment,
\end{abstract}

Luisa Soares-Miranda

soaresmiranda@fade.up.pt

1 Research Center in Physical Activity Health and Leisure, Faculty of Sport, University of Porto, Rua Dr. Plácido Costa 91, 4200-450 Porto, Portugal

2 Gastroenterology Department, Centro Hospitalar São João, Porto, Portugal

3 Department of Surgery, Centro Hospitalar São João, Porto, Portugal

4 Environmental Health Department, National Institute of Public Health, Porto, Portugal

5 EPIUnit, Institute of Public Health, University of Porto, Porto, Portugal

6 Faculty of Nutrition and Food Sciences, University of Porto, Porto, Portugal patients fill in a battery of questionnaires about physical activity, dietary intake, and quality of life, donate blood samples, do physical fitness tests, and use an accelerometer during 7 days. Repeated analyses will be performed to assess changes over time in physical activity, physical fitness, dietary intake, and other factors in relation to recurrence and survival.

Conclusions Results will contribute to highlight the role of physical activity, physical fitness, and nutrition in the quality of life of colorectal cancer survivors, recurrence, and survival. This study will provide important information for policymakers on the potential benefits of future physical activity and nutritional interventions, which are inexpensive, as a way to improve general health of colorectal cancer survivors.

Keywords Cancer survival · Colorectal cancer · Diet . Exercise $\cdot$ Fitness

\section{Background}

Colorectal cancer (CRC) is one of the most common cancers, representing nearly $10 \%$ of the global cancer incidence [1]. In Portugal, CRC incidence ranks second among all cancers, in both men and women after prostate and breast cancers [2], with 5 -year survival rates ranging from 50 to over $60 \%$ [3]. An increase in survival from CRC is expected, not only because of better screening programs but also because treatments have improved. Aging will also lead to a higher number of CRC cases and consequently to a higher number of survivors.

Data on cancer survival are scarce, and there is a need for greater attention on the lifestyle behavior of these survivors [4], especially because cancer diagnosis might be a "window of opportunity" for behavioral change [5]. In USA, more than 
half of patients with cancer die of noncancer causes, being cardiovascular disease (CVD) one of the leading causes [4]. The CVD risk in cancer survivors results of both risk factors and treatment side effects [6]. Physical inactivity and an unhealthy diet that converge as cancer and CVD risk factors, and may have contributed to CRC development in the first place, are regarded as important behavioral targets $[4,6]$. Thus, besides survival, efforts should also start to be directed toward improving the quality of life and health of cancer survivors. With this goal in mind, physical activity (PA) and nutrition might have substantial potential to ameliorate short-term and long-term effects of cancer treatments such as fatigue, body fat gain, and physical fitness (PF) loss, prevent the development of other chronic diseases for which survivors might be at risk such as diabetes and CVD, and to reduce the odds of a recurrence and increase survival.

CRC survivors experience symptoms and adverse effects associated with their cancer and cancer treatment, such as substantial physical and psychosocial impairment that may endure for many years and negatively influence the health, daily functioning, societal participation, and, thus, quality of life of CRC survivors [7-10]. Cancer has the potential to disturb all the aspects of an individual's life; substantial levels of fatigue, anxiety, depression, lower physical and social functioning, chemotherapy-induced peripheral neuropathy, and social, economic, employment, and financial problems have been reported after cancer diagnosis [10-12].

$\mathrm{CRC}$ is, as aforementioned, strongly linked to lifestyle factors, so it is reasonable to assume that those same factors will be important in survivorship to prevent recurrence and to enhance health after cancer treatments. Evidence has been suggesting that the high consumption of red and processed meat, fat, and alcohol accompanied of a low vegetable and fruit consumption as well as low levels of PA and a high body mass index all together may contribute to CRC development [13].

Carcinogenesis is a dynamic and complex process that begins with an irreversible genetic alteration (initiation step) [14]. Although the most widely exploited causes of DNA damage are exogenous factors such as exposure to certain chemicals, genetic alterations may also originate from the decay of the primary structure of DNA, replication errors, and damage due to normal oxidative metabolism and nonenzymatic alkylation from endogeneous metabolites [15]. Actually, cells are constantly exposed to oxidants from normal metabolic reactions in the form of reactive oxygen and nitrogen species [16]. These reactive oxygen species (ROS) are able to oxidize DNA leading to the formation of oxidized bases and single-strand and double-strand breaks. In order to limit the levels of ROS present in cells and therefore the damage they induce, the cell holds many defense mechanisms. Nevertheless, if the production of ROS exceeds the body's natural antioxidant defense mechanisms, cell macromolecules such as DNA, proteins, and lipids may be damaged [17].
Throughout the last years, different authors have demonstrated that metabolism, exercise, and diet are determinants of oxidative DNA damage, often indicated as a biomarker of carcinogenesis [18].

The role of exercise on DNA damage levels seems to depend on the intensity and duration of exercise. Different studies show that intensive (anaerobic physical activity) exercise is based on increased metabolic rate and might thus increase the rate of oxidative DNA [19], while regular exercise, with moderate intensity and duration, upregulates the activity of DNA repair enzymes decreasing the oxidative challenge to the body [20]. A recent study, conducted in healthy individuals, has suggested that higher levels of usual PA are associated with increased levels of DNA repair and unrelated to DNA damage [21].

Diet is a relevant determinant of carcinogenesis as individuals are exposed to a number of carcinogenic and anticarcinogenic substances through edibles [22]. In the case of colon cancer, risk is higher among subjects with dietary patterns characterized by high consumption of red meat and lower among those that predominately consume fruits and vegetables [23].

Deleterious nutritional habits may increase the formation of reactive oxygen species in the intestinal lumen and continuous exposure of the mucosa to these free radicals, which promote oxidative damage to the DNA of the epithelial cells, thereby triggering the appearance of genetic mutations [24].

Altogether, evidence suggests that the mechanism of benefit from diet and physical activity concerns to energy balance, with excess body weight being a risk factor, which is modifiable through lifestyle [25].

Considerably less is known about the role of PA, PF, and eating habits during and after CRC treatments than for its primary prevention [26]. Studies in CRC survivors measuring PA directly, by using accelerometers, are scarce. Moreover, $\mathrm{PF}$, which is essential for daily tasks, has not been comprehensively studied in CRC survivors, and it is also considered to be a very important health outcome and mortality predictor [27]. Besides, there is a need of observational researches to study the associations between PA, PF, and nutrition with quality of life, disease recurrence, and survival by using biological markers, such as DNA damage, that might elucidate mechanism of action, in cancer survivors [28]. Evidencebased lifestyle recommendations are necessary, since the number of CRC survivors is increasing and is expected to rise in the future.

The aim of this study is to uncover the associations of PA, fitness, and nutrition among quality of life, disease recurrence, and survival in CRC survivors by using a multiapproach. We hypothesize that more physical active and fit patients have higher quality of life, less disease recurrence, and higher survival and that a better dietary intake is related with less disease recurrence and higher survival. 


\section{Methods/design}

The CASUS on colorectal patients is a prospective cohort study on PA, fitness, nutrition, and its influences on quality of life, disease recurrence, and survival. The ethics committee of Centro Hospitalar São João, Porto, granted the ethical approval of the study (Porto, Portugal).

\section{Recruitment}

Men and women, above 18 years old, 6 months post-surgery are invited to participate in the study. Stage I-III CRC patients are enrolled and followed up until 2 years after the end of treatment, with repeated measurements performed during preplanned hospital visits at 6,12 , and 24 months post-surgery. Additionally, survival will be assessed 5 years after diagnosis through medical records. Patients with hereditary colorectal cancer disorders (Lynch syndrome, familial adenomatous polyposis, and Peutz-Jeghers syndrome), dementia, or another mental condition that makes them incapable to fill in questionnaires properly are excluded from the study. Two gastroenterologists establish contact with the patients and do the recruitment selection. Patients who agree to participate have to provide written informed consent.

A recruitment rate of, approximately, eight CRC patients per month is expected (100 new participants per year), and a dropout rate of $\sim 20-25 \%$ is expected at each time point.

Since, this study will has several exposures and outcomes, the sample size for this cohort study is based on a relationship of special interest: physical activity and its association with quality of life. Thus, the sample size was based on the estimate that only $5 \%$ of cancer survivors are meeting recommendations for physical activity [29]. A sample size greater than 74 is required to obtain a $95 \%$ confidence interval and a power of $80 \%$. To allow for an expected $60 \%$ response rate to the questionnaire and a dropout rate of about 20-25\%, at least 126 questionnaires should be delivered.

\section{Data collection}

Participants are asked to fill in several questionnaires at 6, 12, and 24 months after surgery (Fig. 1). Additionally, patients are asked to allow a blood sample collection at each time point.

\section{Demographic and health characteristics}

Demographic and health characteristics are evaluated with a self-administered questionnaire, which contains questions on demographics as education, ethnicity, family income per month and occupation, smoking habits, and medication.
Additionally, treatment type and comorbidities are characterized through hospital records.

Health-related quality of life (HRQoL) is assessed with the European Organization for the Research and Treatment of Cancer Quality of Life Questionnaire-Core 30 (EORTC QLQ-C30) [30]. This questionnaire incorporates five functional scales (physical, role, cognitive, emotional, and social), three symptom scales (fatigue, pain, and nausea and vomiting), a global health status and a number of single items assessing additional symptoms commonly reported by cancer patients (dyspnea, loss of appetite, insomnia, constipation, and diarrhea), and perceived financial impact of the disease [31]. This questionnaire is complemented by, a disease-specific module, the CRC-specific CR29 module. Moreover, the patient-reported chemotherapy-induced peripheral neuropathy module (EORTC QLQ-CIPN20) is also used [32, 33]. This questionnaire includes three scales assessing sensory, motor, and autonomic symptoms that can result from neuropathy $[32,33]$.

Health status is assessed with the Portuguese version of the Short Form-36 (SF-36) questionnaire [34, 35]. The SF-36 consists of eight important concepts in health status: physical function, emotional functioning, bodily pain, general health perceptions, vitality, social function, and mental health [34, 35]. The subscales can be combined into the physical and mental component summary scores. All scales will be linearly converted to a $0-100$ scale according to standard scoring procedures, with higher scores indicating better HRQoL.

Fatigue is assessed through the Checklist of Individual Strength (CIS20-P) questionnaire [36]. The CIS20 items represent four dimensions of fatigue: subjective experience of fatigue, concentration, motivation, and PA [36]. Higher scores indicate higher levels of subjective experience of fatigue (ranging from 8 to 56), reduced concentration (5-35), reduced motivation (4-28), and lower levels of PA (3-21). Furthermore, a total CIS20 score (fatigue severity) can be calculated by adding up the scores from each dimension (20-140) [36].

Work productivity and activity impairment questionnaireGeneral Health (WPAI-GH v1.4) is used to measure work productivity losses caused by health problems. The WPAI$\mathrm{GH}$ is composed of six questions that ask the subject if he is currently employed, the number of nonworking hours due to health problems, the number of nonworking hours due to other reasons (e.g., vacation), the number of hours really worked, how the health problems affected his productivity while he was working, and how the health problems affected his daily activities over the last 7 days. The questions are computed according to specific calculation rules and have four scores: (1) percentage of work time missed due to health (absenteeism), (2) percentage of impairment at work due to health (presenteeism), (3) percentage of overall work productivity loss due to health (absenteeism and presenteeism), and (4) 


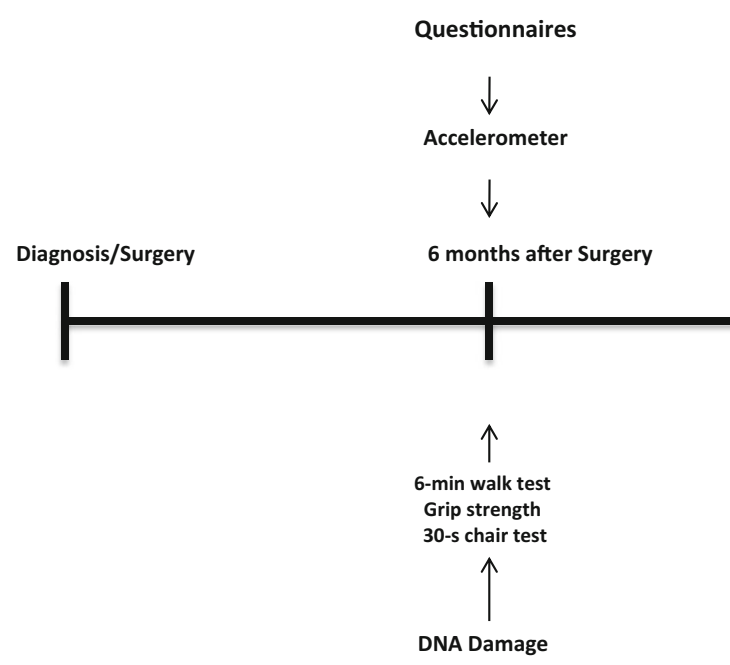

Fig. 1 Overview and design of the CASUS

percentage of daily activity impairment outside of work due to health. High scores indicate prolonged sick leave or impairment and decreased productivity [37].

\section{Physical activity and physical fitness}

PA is assessed by International Physical Activity Questionnaire (IPAQ) and with the use of accelerometer at 6,12 , and 24 months after surgery.

IPAQ short "last week" version is used. IPAQ reports separately vigorous-intensity PA, moderate-intensity PA, and walking in terms of frequency and duration of each specific type of activity, in the past 7 days. This instrument also reports the time spent sitting in an ordinary weekday and weekend day. Both categorical and continuous indicators of PA are possible from the IPAQ short version. Validity and reliability data from 12 countries (including Portugal) have showed that IPAQ has comparable validity and reliability to CSA monitor and to other self-report PA measures (Craig et al. 2003). According to the guidelines for data processing and IPAQ analysis (IPAQ, 2005), total PA can be expressed as MET $\mathrm{min} /$ week (metabolic equivalent), by weighting the reported minutes per week, in each activity category, by the metabolic equivalent specific to each activity.

The Actigraph GT3M accelerometer (Manufacturing Technology, Fort Walton Beach, FL) is used as an objective measure of current daily PA for seven consecutive days. Participants that agree to wear an accelerometer are instructed to use it for seven consecutive days in their right hip by using an adjustable nylon belt. Exceptions have included time spent sleeping and showering. Participants are asked to maintain usual activities. In order for the data to be included in the analyses, participants will need to wear the accelerometer for at least in 4 of the 7 days.
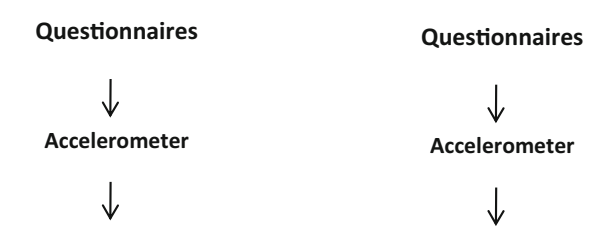

12 months after Surgery

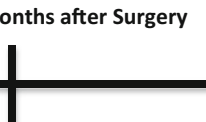

24 months after Surgery
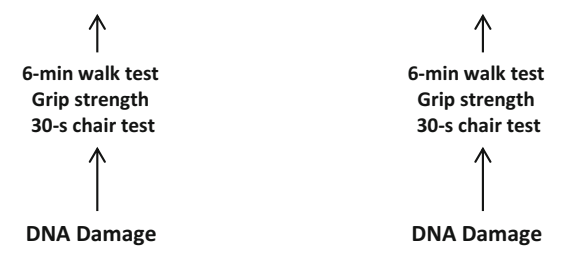

$\mathrm{PF}$ is assessed through the 6-min walk test (6MWT), a 30-s chair-stand test (30sCST), and isometric handgrip strength [38-40]. The 6MWT is performed over a 30-m course in an undisturbed indoor corridor with the turnaround points marked with a cone. Participants are instructed to walk back and forth at their fastest pace covering as much distance as possible during the allotted time. The testing course was standardized, and encouragement is given, and the time remaining is called out every minute. The score is the total distance walked in $6 \mathrm{~min}$, measured in meters. Lower extremity muscle strength is measured by using the $30 \mathrm{sCST}$. Participants are asked to sit in a 45-cm-high chair with arms crossed at the wrists and held against the chest. Participants completed as many "stand ups" as possible within $30 \mathrm{~s}$. The score is the total number of stands executed correctly within $30 \mathrm{~s}$. Grip strength is measured in kilograms in the dominant hand by using a digital hand dynamometer (TKK 5401; Grip-D, Tokyo, Japan) with the subjects in a standing position, with the arms at their side and not touching the body throughout the test. Subjects are instructed to grip maximally but to squeeze only once for each measurement, and three maximal attempts were recorded, with a 30-s rest in between.

\section{Dietary intake}

Dietary intake is assessed by a 7-day food record and a semiquantitative food frequency questionnaire (FFQ). This semiquantitative FFQ was validated for Portuguese adults [41, 14] and designed in accordance with criteria laid out by Willett and adapted to include a variety of typical Portuguese food items [42]. The FFQ administrated at 6 months (baseline) covers the previous 12 months before surgery. The FFQ includes 86 food item and beverage categories, and for each item, the questionnaire offers nine frequency response options, ranging from "never" to "six or more times per day," 
and measures portion size and seasonality. Any foods not covered in the questionnaire are listed by participants in a free-response section. Energy and nutritional intake are estimated with regard to respondents' frequency ratings, portion, and seasonality of each item. Food record includes seven consecutive days (five weekdays and two weekend days) and is completed by participants. Foods' portion size and beverages consumed are estimated by using household measures (cups, glasses, spoons, slices, food wrappers or containers, etc.) as an aid in determining serving sizes. A description of each food and beverage consumed is recorded, including preparation method, time (to the nearest $5 \mathrm{~min}$ ), location, and if appropriate the brand name of the product. Nutrient analysis means that the 7 days will be used in the analysis. Nutrient analysis will be performed by using the software Food Processor SQL (ESHA Research Inc., Salem, OR, USA). This program uses nutritional information from the USA that has been adapted for use with typical Portuguese foods and beverages. The intake of dietary supplements will also be evaluated through interview on the use of multivitamin/minerals/vitamin supplements and on the dosage and intake frequency.

\section{Anthropometric measures}

With participants wearing light clothing and without shoes, body weight is assessed by using a Tanita Inner scan BC532 (Tanita, Hoofddorp, The Netherlands) to the nearest $0.10 \mathrm{~kg}$. Stature is measured by using a stadiometer Seca model 708 (Seca, Hamburg, Germany) to the nearest millimeter. Waist circumference (WC) is measured midway from the lower rib margin to the anterior superior iliac crest by using a nonmetallic tape without significant compression and recorded to $0.1 \mathrm{~cm}$.

\section{DNA damage}

The comet assay is a rapid, simple, visual, and sensitive method assay that can detect DNA damage that is an underlying molecular event driving the initiation and progression of cancer [43].

Briefly, individualized cells are embedded in agarose, lysed with detergent to remove cell and nuclear membranes, and treated with a high salt solution. Nucleoids are formed, containing non-nucleosomal but still supercoiled DNA. Any breaks present in the DNA cause the supercoiling to relax locally, and loops of DNA are then free to extend toward the anode during electrophoresis. At the end of this procedure, damaged cells will resemble comets (hence the name comet assay) while nondamaged cells maintain their round appearance. Comets are viewed by fluorescence microscopy after staining with a suitable fluorescent DNA-binding dye [44].
Throughout the years, there were several modifications and innovations to the original protocol which led to an array of comet assay variants that detect different types of DNA alterations. Specific types of DNA lesions, related to oxidative damage, can be measured by using lesion-specific repair enzymes [45].

To detect basal levels of DNA damage, we will run the alkaline comet assay according to what has previously described by Costa et al. [46]. In addition, the assay will also be performed with an additional step of incubation with the restriction enzymes formamidopyrimidine-DNA glycosylase (FPG) and 8-oxoguanine DNA $N$-glycosylase 1 (hOGG1) to detect oxidative damage. FPG is specific for oxidized purines, including 8-oxo-7,8-dihydroguanine (8-oxoGua), 2,6diamino-4-hydroxy-5-formamidopyrimidine (FaPyGua), and 4,6-diamino-5-formamidopyrimidine, (FaPyAde) and other ring-opened purines, whereas hOGG1 is specific for 8oxoGua and methyl FapyGua (MeFapyGua) [45].

The size and shape of the comet and the distribution of DNA within the comet correlate with the extent of DNA damage present in the individual cell. Images will be captured by an online CCD camera and analyzed with the Comet Assay IV analysis software (Perceptive Instruments). For each cell, DNA damage will be expressed as the percentage of DNA in the comet tail. A total of 100 cells are analyzed per sample, 50 per duplicate slide.

\section{Recurrence and survival}

According to the hospital internal protocol, for colorectal cancer, the follow-up is the following: A colonoscopy will be done 3 months after surgery and then annually until 5 years after diagnosis and then every 2 years; an ultrasound and carcinoembryonic antigen (CAE) determination will be done every 6 months. These results will be used to determine recurrence. Survival will be assessed throughout medical records.

\section{Endpoints}

The primary endpoints will be disease recurrence and death. Secondary endpoint will be quality of life, fatigue, and work productivity.

\section{Nonparticipants and subjects lost in follow-up}

Comparisons to determine whether demographic and/or cancer-related characteristics were differed between participants and nonparticipants will be made. Additionally, comparisons between those who completed the baseline 
questionnaire and those who were lost to follow-up will be made to determine possible differences.

Participants may withdraw from the study at anytime; in these circumstances, we will use the already collected data from those participants. Participants' missing information might be dealt with multiple imputations since imputation adjusts for differences among nonrespondents and respondents on existent variables for both and incorporated in the imputation procedure, as well as differences on variables not integrated in the model that are predicted by the model [47]. Statistical analysis will be done with and without the imputations to observe if results are maintained and reported in results.

\section{Conclusions}

The CASUS on colorectal patients will critically assess important research questions, which may provide new leads for future intervention studies and improved lifestyle counseling strategies on CRC. This study will provide important information for policymakers on the potential benefits of future PA and nutritional interventions, which are inexpensive, as a way to improve general health of CRC survivors.

Acknowledgments Luisa Soares-Miranda is supported by the grant SFRH/BPD/76947/2011 funded by FCT (QREN-POPH-Type 4.1Advanced Training, subsidized by the European Social Fund and national funds of MEC), PTDC/DES/099018/2008-FCT/FCOMP-01-0124FEDER-009573, and the Research Centre in Physical Activity Health and Leisure is supported by FCT: UID/DTP/00617/2013. Carla Costa is supported by the grant SFRH/BPD/96196/2013, funded by FCT (QRENPOPH-Type 4.1-Advanced Training, subsidized by the European Social Fund and national funds of MEC).

\section{References}

1. May MT, Ingle SM, Costagliola D, Justice AC, de Wolf F, Cavassini M, D’Arminio Monforte A, Casabona J, Hogg RS, Mocroft A, Lampe FC, Dabis F, Fatkenheuer G, Sterling TR, del Amo J, Gill MJ, Crane HM, Saag MS, Guest J, Brodt HR, Sterne JA, Antiretroviral Cohort C (2014) Cohort profile: antiretroviral therapy cohort collaboration (ART-CC. Int J Epidemiol 43(3): 691-702. doi:10.1093/ije/dyt010

2. Pinto CG, Paquete AT, Pissarra I (2010) Colorectal cancer in Portugal. The European journal of health economics : HEPAC : health economics in prevention and care 10 Suppl 1:S65-S73. doi:10.1007/s10198-009-0187-9

3. De Angelis R, Sant M, Coleman MP, Francisci S, Baili P, Pierannunzio D, Trama A, Visser O, Brenner H, Ardanaz E, Bielska-Lasota M, Engholm G, Nennecke A, Siesling S, Berrino F, Capocaccia R, Group E-W (2014) Cancer survival in Europe 1999-2007 by country and age: results of EUROCARE - 5-a population-based study. The Lancet Oncology 15(1):23-34. doi:10.1016/S1470-2045(13)70546-1

4. Lajous M, Mozaffarian D, Mozaffarian R, Schrag D, Adami HO (2011) Lifestyle prescriptions for cancer survivors and their communities. J Intern Med 269(1):88-93. doi:10.1111/j.13652796.2010.02273.x

5. Moller T, Lillelund C, Andersen C, Ejlertsen B, Norgaard L, Christensen KB, Vadstrup E, Diderichsen F, Hendriksen C, Bloomquist K, Adamsen L (2013) At cancer diagnosis: a 'window of opportunity' for behavioural change towards physical activity. A randomised feasibility study in patients with colon and breast cancer. BMJ Open 3(11):e003556. doi:10.1136/bmjopen-2013003556

6. Wolin KY, Colditz GA (2011) Implementing chronic disease prevention amongst cancer survivors. J Intern Med 269(1):85-87. doi:10.1111/j.1365-2796.2010.02295.x

7. Jansen L, Herrmann A, Stegmaier C, Singer S, Brenner H, Arndt V (2011) Health-related quality of life during the 10 years after diagnosis of colorectal cancer: a population-based study. Journal of clinical oncology : official journal of the American Society of Clinical Oncology 29(24):3263-3269. doi:10.1200 /jco.2010.31.4013

8. Jansen L, Koch L, Brenner H, Arndt V (2010) Quality of life among long-term $(>/=5$ years $)$ colorectal cancer survivors - systematic review. European journal of cancer (Oxford, England : 1990) 46(16): 2879-2888. doi:10.1016/j.ejca.2010.06.010

9. Mosher CE, Sloane R, Morey MC, Snyder DC, Cohen HJ, Miller PE, Demark-Wahnefried W (2009) Associations between lifestyle factors and quality of life among older long-term breast, prostate, and colorectal cancer survivors. Cancer 115(17):4001-4009. doi: $10.1002 /$ cncr.24436

10. Phipps E, Braitman LE, Stites S, Leighton JC (2008) Quality of life and symptom attribution in long-term colon cancer survivors. J Eval Clin Pract 14(2):254-258. doi:10.1111/j.13652753.2007.00842.x

11. Harrington CB, Hansen JA, Moskowitz M, Todd BL, Feuerstein M (2010) It's not over when it's over: long-term symptoms in cancer survivors - a systematic review. Int J Psychiatry Med 40(2):163181

12. Alfano CM, Rowland JH (2006) Recovery issues in cancer survivorship: a new challenge for supportive care. Cancer J 12(5):432443

13. Caswell S, Anderson AS, Steele RJ (2008) Diet and physical activity in patients with colorectal adenomas: directions for intervention programmes. Journal of human nutrition and dietetics : the official journal of the British Dietetic Association 21(5):494-501. doi:10.1111/j.1365-277X.2008.00884.x

14. Weston A, Harris CC (2003) Chemical carcinogenesis. In: Kufe DW, Holland JF (eds) Cancer medicine. Hamilton, Ont., Canada

15. Friedberg EC, McDaniel LD, Schultz RA (2004) The role of endogenous and exogenous DNA damage and mutagenesis. Current Opinion in Genetics \& Development 14(1):5-10. doi:10.1016/j. gde.2003.11.001

16. Loft S, Poulsen HE (1996) Cancer risk and oxidative DNA damage in man. J Mol Med (Berl) 74(6):297-312

17. De Bont R, van Larebeke N (2004) Endogenous DNA damage in humans: a review of quantitative data. Mutagenesis 19(3):169-185

18. Valko M, Izakovic M, Mazur M, Rhodes CJ, Telser J (2004) Role of oxygen radicals in DNA damage and cancer incidence. Mol Cell Biochem 266(1-2):37-56

19. Hartmann A, Plappert U, Raddatz K, Grunert-Fuchs M, Speit G (1994) Does physical activity induce DNA damage? Mutagenesis 9(3):269-272

20. Radak Z, Chung HY, Koltai E, Taylor AW, Goto S (2008) Exercise, oxidative stress and hormesis. Ageing Res Rev 7(1):34-42. doi:10.1016/j.arr.2007.04.004

21. Cash SW, Beresford SA, Vaughan TL, Heagerty PJ, Bernstein L, White E, Neuhouser ML (2014) Recent physical activity in relation to DNA damage and repair using the comet assay. J Phys Act Health 11(4):770-776. doi:10.1123/jpah.2012-0278 
22. Tseng M, Satia JA (2010) 14.21—diet and cancer. In: McQueen CA (ed) Comprehensive toxicology, Second edn. Elsevier, Oxford, pp. 433-448. doi:10.1016/B978-0-08-046884-6.01422-6

23. Magalhaes B, Peleteiro B, Lunet N (2012) Dietary patterns and colorectal cancer: systematic review and meta-analysis. Eur J Cancer Prev 21(1):15-23. doi:10.1097/CEJ.0b013e3283472241

24. Sesink AL, Termont DS, Kleibeuker JH, Van der Meer R (1999) Red meat and colon cancer: the cytotoxic and hyperproliferative effects of dietary heme. Cancer Res 59(22):5704-5709

25. Davies NJ, Batehup L, Thomas R (2011) The role of diet and physical activity in breast, colorectal, and prostate cancer survivorship: a review of the literature. Br J Cancer 105 Suppl 1:S52-S73. doi:10.1038/bjc.2011.423

26. Winkels RM, Heine-Broring RC, van Zutphen M, van HartenGerritsen S, Kok DE, van Duijnhoven FJ, Kampman E (2014) The COLON study: colorectal cancer: longitudinal, observational study on nutritional and lifestyle factors that may influence colorectal tumour recurrence, survival and quality of life. BMC Cancer 14:374. doi:10.1186/1471-2407-14-374

27. Garber CE, Blissmer B, Deschenes MR, Franklin BA, Lamonte MJ, Lee IM, Nieman DC, Swain DP, American College of Sports M (2011) American College of Sports Medicine position stand. Quantity and quality of exercise for developing and maintaining cardiorespiratory, musculoskeletal, and neuromotor fitness in apparently healthy adults: guidance for prescribing exercise. Med Sci Sports Exerc 43(7):1334-1359. doi:10.1249/MSS.0b013e318213fefb

28. Ballard-Barbash R, Friedenreich CM, Courneya KS, Siddiqi SM, McTiernan A, Alfano CM (2012) Physical activity, biomarkers, and disease outcomes in cancer survivors: a systematic review. J Natl Cancer Inst 104(11):815-840. doi:10.1093/jnci/djs207

29. Smith WA, Nolan VG, Robison LL, Hudson MM, Ness KK (2011) Physical activity among cancer survivors and those with no history of cancer - a report from the National Health and nutrition examination survey 2003-2006. Am J Transl Res 3(4):342-350

30. Aaronson NK, Ahmedzai S, Bergman B, Bullinger M, Cull A, Duez NJ, Filiberti A, Flechtner H, Fleishman SB, de Haes JC et al (1993) The European Organization for Research and Treatment of cancer QLQ-C30: a quality-of-life instrument for use in international clinical trials in oncology. J Natl Cancer Inst 85(5):365-376

31. Fayers P AN, Bjordal K, Groenvold M, Curran D, Bottomley A, on behalf of the European Quality of Life Group: (2001) The EORTC QLQ-C30 scoring manual. In 3rd edition.

32. Postma TJ, Aaronson NK, Heimans JJ, Muller MJ, Hildebrand JG, Delattre JY, Hoang-Xuan K, Lanteri-Minet M, Grant R, Huddart R, Moynihan C, Maher J, Lucey R, Group EQoL (2005) The development of an EORTC quality of life questionnaire to assess chemotherapy-induced peripheral neuropathy: the QLQ-CIPN20. Eur J Cancer 41(8):1135-1139. doi:10.1016/j.ejca.2005.02.012
33. Lavoie Smith EM, Barton DL, Qin R, Steen PD, Aaronson NK, Loprinzi CL (2013) Assessing patient-reported peripheral neuropathy: the reliability and validity of the European Organization for Research and Treatment of cancer QLQ-CIPN20 questionnaire. Quality of life research : an international journal of quality of life aspects of treatment, care and rehabilitation 22(10):2787-2799. doi:10.1007/s11136-013-0379-8

34. Ferreira PL (2000) Development of the Portuguese version of MOS SF-36. Part I. Cultural and linguistic adaptation. Acta medica portuguesa 13(1-2):55-66

35. Ferreira PL (2000) Development of the Portuguese version of MOS SF-36. Part II - validation tests. Acta medica portuguesa 13(3): 119-127

36. Marques MDGV, Gouveia MJ, Cordeiro A, Leal I, Maes S (2013) Psychometric properties of the Portuguese version of the checklist of individual strength (CIS20-P). Psychology, Community \& Health 2(1):11-18

37. ASSOCIATES R (2014) http://www.reillyassociates.net/WPAI Translations.html

38. Rikli RE, Jones CJ (1999a) Development and validation of a functional fitness test for community-residing older adults. J Aging Phys Act 6:127-159

39. Schmidt K, Vogt L, Thiel C, Jager E, Banzer W (2013) Validity of the six-minute walk test in cancer patients. Int J Sports Med 34(7): 631-636. doi:10.1055/s-0032-1323746

40. American Thoracic S, American College of Chest P (2003) ATS/ ACCP statement on cardiopulmonary exercise testing. Am J Respir Crit Care Med 167(2):211-277. doi:10.1164/rccm.167.2.211

41. Lopes C, Aro A, Azevedo A, Ramos E, Barros H (2007) Intake and adipose tissue composition of fatty acids and risk of myocardial infarction in a male Portuguese community sample. J Am Diet Assoc 107(2):276-286. doi:10.1016/j.jada.2006.11.008

42. Willett W (1998) Food frequency methods in nutritional epidemiology. Oxford University Press, New York

43. McKenna DJ, McKeown SR, McKelvey-Martin VJ (2008) Potential use of the comet assay in the clinical management of cancer. Mutagenesis 23(3):183-190

44. Collins AR, Dobson VL, Dusinska M, Kennedy G, Stetina R (1997) The comet assay: what can it really tell us? Mutat Res 375(2):183-193

45. Smith CC, O'Donovan MR, Martin EA (2006) hOGG1 recognizes oxidative damage using the comet assay with greater specificity than FPG or ENDOIII. Mutagenesis 21(3):185-190. doi:10.1093 /mutage/gel019

46. Costa S, Coelho P, Costa C, Silva S, Mayan O, Santos LS, Gaspar J, Teixeira JP (2008) Genotoxic damage in pathology anatomy laboratory workers exposed to formaldehyde. Toxicology 252:40-48

47. Raghunathan TE (2004) What do we do with missing data? Some options for analysis of incomplete data. Annu Rev Public Health 25:99-117. doi:10.1146/annurev.publhealth.25.102802.124410 